\title{
Glucocorticoid-induced leucine zipper regulates liver fibrosis by suppressing CCL2-mediated leukocyte recruitment
}

\author{
Sara Flamini ${ }^{1,2}$, Philipp Sergeev $\mathbb{B}^{3,4}$, Zenobio Viana de Barros ${ }^{1}$, Tommaso Mello $\mathbb{1}^{5}$, Michele Biagioli ${ }^{1}$,

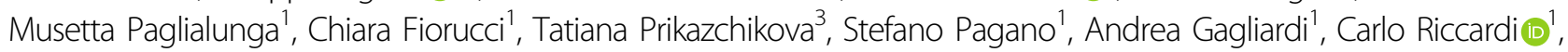

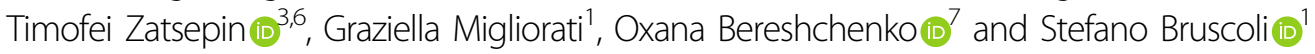

\begin{abstract}
Liver fibrosis (LF) is a dangerous clinical condition with no available treatment. Inflammation plays a critical role in LF progression. Glucocorticoid-induced leucine zipper (GILZ, encoded in mice by the Tsc22d3 gene) mimics many of the anti-inflammatory effects of glucocorticoids, but its role in LF has not been directly addressed. Here, we found that GILZ deficiency in mice was associated with elevated CCL2 production and pro-inflammatory leukocyte infiltration at the early LF stage, resulting in enhanced LF development. RNA interference-mediated in vivo silencing of the CCL2 receptor CCR2 abolished the increased leukocyte recruitment and the associated hepatic stellate cell activation in the livers of GILZ knockout mice. To highlight the clinical relevance of these findings, we found that TSC22D3 mRNA expression was significantly downregulated and was inversely correlated with that of CCL2 in the liver samples of patients with LF. Altogether, these data demonstrate a protective role of GILZ in LF and uncover the mechanism, which can be targeted therapeutically. Therefore, modulating GILZ expression and its downstream targets represents a novel avenue for pharmacological intervention for treating LF and possibly other liver inflammatory disorders.
\end{abstract}

\section{Introduction}

Liver fibrosis (LF) is characterized by excessive scar tissue formation during liver repair. LF is associated with most chronic liver diseases, including viral infections, alcoholic liver cirrhosis, nonalcoholic fat liver disease (NAFLD), and autoimmune hepatitis. Although asymptomatic, LF represents a dangerous medical condition leading to irreversible and potentially lethal liver cirrhosis ${ }^{1,2}$. Understanding the molecular regulatory mechanisms in

\footnotetext{
Correspondence: Oxana Bereshchenko (oxana.bereshchenko@unipg.it) 'Department of Medicine and Surgery, University of Perugia, Severi Place 1, 06132 Perugia, Italy

'Laboratory of Immune Regeneration and Experimental Hematology,

Department of Pediatric Hematology and Oncology, Bambino Gesù Children Hospital, Viale San Paolo 15, Roma 00146, Italy

Full list of author information is available at the end of the article

These authors contributed equally: Oxana Bereshchenko, Stefano Bruscoli

Edited by M. Agostini
}

the fibrotic processes is crucial for developing effective preventive and therapeutic strategies.

A complex interplay among different cell types regulates LF. The replacement of hepatocytes damaged by hepatotoxic agents, e.g., hepatitis viruses, alcohol metabolites, bile acids, and some pharmacologic agents, activates an inflammatory response and white blood cell recruitment in the earliest stages following liver injury ${ }^{3}$. Inflammation plays an important role in the development of virtually all forms of fibrosis ${ }^{4}$. Both innate and adaptive immunity cells are found in lesions at the early stages of LF development, and include monocytes/macrophages, natural killer/natural killer $\mathrm{T}(\mathrm{NK} / \mathrm{NKt})$ cells, and $\mathrm{T}$ and $\mathrm{B}$ lymphocytes ${ }^{3}$. Infiltrating immune cells produce pro-inflammatory and pro-fibrotic cytokines and chemokines, leading to the activation of hepatic stellate cells (HSCs), fibroblasts, and myofibroblasts, which proliferate and produce large amounts of

\section{(c) The Author(s) 2021}

(c) (i) Open Access This article is licensed under a Creative Commons Attribution 4.0 International License, which permits use, sharing, adaptation, distribution and reproduction cc) in any medium or format, as long as you give appropriate credit to the original author(s) and the source, provide a link to the Creative Commons license, and indicate if changes were made. The images or other third party material in this article are included in the article's Creative Commons license, unless indicated otherwise in a credit line to the material. If material is not included in the article's Creative Commons license and your intended use is not permitted by statutory regulation or exceeds the permitted use, you will need to obtain permission directly from the copyright holder. To view a copy of this license, visit http://creativecommons.org/licenses/by/4.0/. 
extracellular matrix components ${ }^{3}$. The fine balance between specific cytokines produced by resident and infiltrating macrophages and $\mathrm{T}$ and NK cells defines the resolution or enhancement of inflammatory and fibrotic processes. Cytokines that promote fibrosis include TGF$\beta 1$, IL-6, TNF- $\alpha$, and IL-1, produced by activated macrophages, and IL-4 and IL-13, associated with the T helper type 2 (Th2) response ${ }^{1,5,6}$. While it is not possible to clearly distinguish between the relative contribution of the innate and adaptive immune systems to inflammatory response propagation in LF development, identifying the factors regulating leukocyte recruitment to the liver provides insight into the mechanisms that restrain LF.

Chemokines regulate circulating immune cell migration and activity during fibrosis development. Monocyte chemoattractant proteins (MCPs) are potent chemoattractants of monocytes, activated $\mathrm{T}$ cells, NK cells, and immature dendritic cells ${ }^{7}$. MCP-1 (or CCL2) binds to its receptor (CCR2); besides recruiting leukocytes, it also directly influences fibrogenic HSC activity. Experimental models using CCL2- or CCR2-deficient mice have demonstrated the functional relevance of the CCL2-CCR2 pathway in $\mathrm{LF}^{3,7-11}$. Therefore, strategies that dampen excessive inflammatory responses and immune cell recruitment to the liver represent therapeutic potential for LF management.

Glucocorticoids (GCs) are potent anti-inflammatory drugs used for treating a wide spectrum of inflammatory diseases ${ }^{12-14}$, including those of the liver, e.g., severe alcoholic hepatitis ${ }^{15}$, autoimmune hepatitis ${ }^{16}$, and liver failure $^{17,18}$. Their efficacy in treating LF is attributed to their ability to inhibit leukocyte migration to inflammation sites and suppress pro-inflammatory mediator production. Treating mice with the synthetic GC prednisolone suppressed inflammatory responses in a model of carbon tetrachloride $\left(\mathrm{CCl}_{4}\right)$-induced hepatitis, but surprisingly exacerbated liver injury and delayed liver repair ${ }^{19}$. GCs have a controversial effect on LF because they exert opposing effects on immune cells and on $\mathrm{HSCs}^{20}$. Overall, the serious adverse effects of GC therapy represent the limiting factor to their prolonged usage for treating liver inflammation. Therefore, there is a strong need to identify means of disassociating the antiinflammatory and metabolic effects of classical GCs to improve the therapy of liver diseases, including LF.

Glucocorticoid-induced leucine zipper (Gilz or $T s c 22 d 3)$ is a gene rapidly induced by GCs in T cells ${ }^{21,22}$. GILZ functions in immune ${ }^{21,22}$ and nonimmune cells, including germ ${ }^{23}$, muscle ${ }^{24-26}$, and endothelial cells ${ }^{27}$ and adipocytes ${ }^{28}$. GILZ mimics the effects of GCs in many cellular processes regulating apoptosis ${ }^{29,30}$, and cell activation and differentiation ${ }^{21,31-39}$. These effects are partly dependent on the ability of GILZ to repress the ERK-MAPK-NF- $\mathrm{KB}$ pathways important for leukocyte activation, migration, and pro-inflammatory cytokine and chemokine expression ${ }^{40-43}$. The immunomodulatory effects of GILZ have been demonstrated in in vivo models of inflammation ${ }^{35,44}$.

The role of GILZ in LF development has not been directly investigated. Deregulated GILZ expression was evidenced in autoimmune hepatitis patients ${ }^{45}$, and the GC receptor-GILZ pathway was implicated in regulating liver inflammation in obese mice ${ }^{46}$. We addressed the role of GILZ in LF development in a mouse model of $\mathrm{CCl}_{4}$-induced $\mathrm{LF}^{23}$. We found that GILZ deficiency in $T s c 22 d 3$ knockout (hereafter: GILZ KO) mice is associated with more pronounced LF, characterized by increased CCL2 expression and leukocyte liver infiltration. Downregulation of CCR2 expression via RNA interference (RNAi)-mediated silencing in vivo reverted the enhanced leukocyte recruitment in GILZ KO mice and the associated HSC activation. Moreover, human gene expression data analysis revealed that TSC22D3 mRNA expression is downregulated in NAFLD patients with LF and correlates inversely with that of CCL2. Therefore, our data show that GILZ restrains LF development by controlling CCL2-dependent leukocyte trafficking into the liver, and that this pathway can be targeted therapeutically to restrain LF development and/ or progression.

\section{Materials and methods \\ Mice}

Mice were kept in Center for Preclinical Studies of the University of Perugia and treated according to the Italian (D.M. 116.192), European (Official Journal of European Community 358/1, December 18, 1986), and American (Animal Welfare Assurance No. A5594-01, Department of Health and Human Services, Washington, DC) laws, approved by University of Perugia. Male C57BL/6 GILZ $\mathrm{KO}$ and wild-type (WT) littermate mice were generated, as previously described ${ }^{23}$. Mice overexpressing GILZ (GILZ transgenic, GILZ TG) were previously described ${ }^{47}$.

\section{Liver fibrosis induction}

$\mathrm{LF}$ was induced by $\mathrm{CCl}_{4}$ administration (FLUKA, Cod. No. 87030) according to a previously published method ${ }^{48}$. For this purpose, the mice were administered i.p. $500 \mu \mathrm{L} / \mathrm{Kg}$ body weight of $\mathrm{CCl}_{4}$ in an equal volume of olive oil.

\section{Tissue histology}

Portions of the right and left liver lobes were fixed in $10 \%$ formalin, embedded in paraffin, sectioned, and stained with Sirius red for morphometric analysis.

\section{Immunohistochemistry}

Paraffin-embedded tissue sections $7 \mu \mathrm{m}$ thick were rehydrated, boiled in Tris/EDTA $\mathrm{pH}=9$ for $20 \mathrm{~min}$, 
incubated in $3 \% \mathrm{H}_{2} \mathrm{O}_{2}$ for $10 \mathrm{~min}$ to inhibit endogenous peroxidases, and then incubated with a blocking solution (2.5\% normal goat serum, Vector Laboratories). Monoclonal anti- $\alpha$-SMA (clone 1A4, cat. A2547 Sigma-Aldrich, dil. 1:200) was incubated overnight at $4^{\circ} \mathrm{C}$ in blocking solution. The secondary antibody anti-Mouse IgG ImmPRESS Reagent Kit (Vector Laboratories, cat. MP7402) was incubated for $30 \mathrm{~s}$ at RT and the chromogenic reaction developed with DAB (Sigma-SIGMAFAST ${ }^{\mathrm{TM}}$ DAB). Slides were then counter-colored with hematoxylin (Gill's 3, Bio-Optica), dehydrated, and mounted in DPX.

Frozen tissue was sectioned ( $7 \mu \mathrm{m}$ thick), air dried, and fixed in cold acetone $\left(-20^{\circ} \mathrm{C}\right)$ for $20 \mathrm{~min}$. Slides were then air dried and kept in $0.3 \% \mathrm{H}_{2} \mathrm{O}_{2}$ in methanol for $30 \mathrm{~min}$ to inhibit endogenous peroxidases. Following $2.5 \%$ NGS blocking, anti- $\alpha$-SMA was incubated overnight at $4^{\circ} \mathrm{C}$ at 1:500 dilution. The secondary antibody anti-mouse IgG ImmPRESS Reagent Kit (Vector Laboratories, cat.MP7402) was incubated for $30 \mathrm{~s}$ at RT and the chromogenic reaction developed with $D A B$ (Sigma-SIGMAFAST $^{\mathrm{TM}}$ DAB with metal Enhancer). Section was counterstained with Nuclear Fast Red (Vector Laboratories), dehydrated, and mounted in DPX (cat. 06522, Sigma -Aldrich).

\section{RNA isolation and qPCR}

RNA was isolated from whole tissue fragment using RNA-XPress $^{\mathrm{TM}}$ Reagent (MB601, HIMEDIA) and reversetranscribed using PrimeScript RT reagent Kit, with gDNA Eraser (Perfect Real-Time-TAKARA). Quantitative real-time PCR (qPCR) was performed using the 7300 Real-Time PCR System (Applied Biosystems), SYBR ${ }^{\mathrm{TM}}$ Select Master Mix (Applied Biosystems), and TaqMan ${ }^{\mathrm{TM}}$ Gene Expression Master Mix(Applied Biosystems). The qPCR TaqMan probes (Applied Biosystems) were as follows: Fas, Mm01204974; FasL, Mm00438864; Bcl-xL, Mm00437783; and Actb, 4352341E. Primers using in amplification using Sybergreen methods are listed in Supplementary Table 2.

\section{Isolation of liver infiltrating leukocytes}

The harvested livers were digested by liver dissociation kit (Miltenyi Biotec), following manufacturer's instruction. Briefly, homogenized livers were incubated with dissociation mix for $45 \mathrm{~min}$, the cell suspensions were applied to a $100 \mu \mathrm{m}$ and then $70 \mu \mathrm{m}$ cell strainer. Released cells were layered on a $40-80 \%$ Percoll gradient and spun at $400 \times g$ for $20 \mathrm{~min}$ to get an enriched LPMC population and processed as described ${ }^{49}$.

\section{Isolation of liver cells}

Primary hepatocytes and Kupffer cells were isolated via a two-step collagenase liver perfusion. Briefly, mice were anesthetized with ketamine/xylazine, and perfused via the portal vein first with Liver Perfusion Medium (Gibco cat. 17701038) to remove the blood and then with a collagenase solution (cat. C5138 Sigma-Aldrich, 0.5\% w/v in minimum essential medium). The cell suspension was filtered through a $70 \mu \mathrm{m}$ cell strainer (Greiner Bio-one) and centrifuged to separate hepatocytes (pellet) from nonparenchymal cells (supernatant). Pelleted hepatocytes were purified through centrifugation over $40 \%$ Percoll and plated in a collagen sandwich, while Kupffer cells were purified from the non-parenchymal cells pool by differential adhesion on plastic. RNA from purified hepatocytes and $\mathrm{KC}$ was extracted $24 \mathrm{~h}$ after plating.

\section{Antibodies and flow cytometry}

Cells were prepared and stained with directly conjugated antibodies as described ${ }^{50}$. Monoclonal antibodies were purchased from Thermo Fisher: anti-CD3e (1452C11), anti-CD4 (GK1.5), anti-CD8 (53-6.7), anti-CD11b (M1/70), anti-B220 (RA3-6B2), anti-CD49b (DX5), and anti-Gr1 (RB6-8C5). Analyses were performed using the ATTUNE NxT three-laser standard configuration (Life Technologies), and data were analyzed using Flow Jo software (Tree Star).

\section{siRNA description and LNP formulation}

We used one small interfering RNA (siRNA) described by Leuschner et al. ${ }^{51}$ and in Supplementary Methods. siRNA was selected to avoid off-target activity based on several known criteria as described previously ${ }^{52}$. siRNA modification with $2^{\prime}$-OMe pyrimidine nucleotides and $3^{\prime}$-internucleotide phosphorothioates should reduce immune response and increase stability in vivo. Potency of siRNA targeting CCR2 were studied by transfection in Hepa1-6 cells followed by qPCR analysis after $24 \mathrm{~h}$. siRNA were formulated in lipid nanoparticles (LNPs), as previously described ${ }^{52}$. Particle size measurements were performed using a Zetasizer Nano ZSP (Malvern Panalytical) according to the manufacturer's protocol, siRNA entrapment efficiency was determined using the QuantiT $^{\mathrm{TM}}$ RiboGreen ${ }^{\circledR}$ reagent (R11491, Thermo Fisher Scientific) as described ${ }^{52}$.

\section{Analysis of gene expression using available gene expression data}

Transcriptional profile of NASH patients was downloaded from Gene Expression Omnibus (GEO) with accession number GSE48452. We analyzed total RNAseq data from 64 human liver samples grouped into "control" (healthy individuals; $n=14)$, "healthy obese" $(n=27)$, "NASH-high fat score" (patients presenting NASH and with a fat score equal to or higher than $70 ; n=9$ ), and "NASH-fibrosis 1-4" (patients with a fibrosis score ranging from 1 to $4 ; n=14$ ). Transcriptional profile of NAFLD patients was downloaded from the GEO with 
accession number GSE49541. This dataset represents two clinically defined pathological groups at the extremes of NAFLD: mild NAFLD (fibrosis stages $0-1 ; n=40$ ), with little risk of developing severe liver disease; advanced NAFLD (fibrosis stages $3-4 ; n=32$ ), with significant likelihood of developing liver-related morbidity and mortality. Dataset passed through Kolmogorov-Smirnov test, and statistical analysis was performed by parametric ANOVA test for multiple comparisons and or Mann-Whitney test using GraphPad Prism 6.0 software. Pearson's correlation coefficient for gene expression correlation $(R)$ and Tukey's multiple comparison test were performed in GraphPad Prism 6.0.

\section{Statistical analysis}

Statistical analysis was performed with Prism 6.0 (GraphPad). The two-tailed unpaired Student $t$ test or nonparametric Mann-Whitney $U$ test was used for statistical comparisons. For multiple comparisons, two-way ANOVA was performed.

\section{Results}

GILZ deficiency is associated with enhanced LF development in mice

GILZ is expressed both in hepatocytes and in Kuppfer cells (Fig. S1). To evaluate possible contribution of GILZ in the development of LF, WT, and GILZ KO mice were treated with $\mathrm{CCl}_{4}$ for 6-7 weeks to induce $\mathrm{LF}$ as described ${ }^{48}$. The degree of LF development upon $\mathrm{CCl}_{4}$-induced chronic liver injury was examined evaluating the degree of collagen deposition in the livers of WT and GILZ KO mice using Sirius Red staining. We found that the GILZ KO mice showed increased collagen deposition in the livers, following $\mathrm{CCl}_{4}$ treatment compared to similarly treated WT mice (Fig. 1A, B). To investigate whether the absence of GILZ is associated with compromised liver function following $\mathrm{CCl}_{4}$-incurred damage, serum levels of markers of liver damage, aspartate aminotransferase (AST), and alanine aminotransferase $(\mathrm{ALT})^{53}$, were evaluated in WT and GILZ KO mice treated for 6-7 weeks with $\mathrm{CCl}_{4}$. The AST serum levels were significantly increased in GILZ KO mice upon $\mathrm{CCl}_{4}$ treatment compared to similarly treated WT mice (Fig. 1C), while the ALT serum levels showed a tendency increase in GILZ KO mice compared to similarly treated WT mice (Fig. 1D). In addition, Liver Index (LI), representing the ratio of liver weight and body weight $x$ 1000 (ref. ${ }^{54}$ ), was also significantly increased in GILZ KO mice following $\mathrm{CCl}_{4}$ treatment compared to similarly treated WT mice (Fig. 1E). Since the increase in $\alpha$-smooth muscle antigen ( $\alpha$-SMA) expression characterizes active fibrosis process, we compared the level of mRNA expression of Asma in livers isolated from WT and GILZ KO mice treated with $\mathrm{CCl}_{4}$ for 7 weeks. As expected, Asma mRNA expression was significantly increased in both WT and GILZ KO mice following $\mathrm{CCl}_{4}$ treatment; however, Asma levels were significantly higher in GILZ KO compared to similarly treated WT mice (Fig. 1F). Taken together, these data demonstrate that the absence of GILZ results in an enhanced development of experimentally induced LF in mice, and suggest that GILZ plays a role in LF development process.

\section{GILZ deficiency is associated with elevated HSC activation}

HSC play a key role in the fibrotic process following liver injury ${ }^{55,56}$. $\alpha$-SMA expression serves as a marker of HSCs in normal and pathologic liver tissue. To demonstrate that enhanced $\mathrm{CCl}_{4}$-induced $\mathrm{LF}$ development observed in GILZ KO mice is associated with HSC activation, we performed immunohistochemistry analysis of $\alpha$-SMA expression in livers isolated from WT and GILZ $\mathrm{KO}$ mice treated with oil (control) or $\mathrm{CCl}_{4}$ (Fig. 2). The data show that the absence of GILZ is associated with increased expression of $\alpha$-SMA and HSC expansion in the pericentral zone of the liver lobule, consistent with $\mathrm{CCL}_{4}$ induced damage pattern, both at acute (Fig. 2A) and chronic (Fig. 2B) phases of LF development. These results show that the absence of GILZ is associated with elevated expression of $\alpha$-SMA during LF development, suggesting that GILZ restrains HSC activation.

\section{GILZ deficiency results in increased leukocytes recruitment in liver upon $\mathrm{CCl}_{4}$ treatment}

To investigate the mechanisms underlying the enhanced LF development in GILZ KO mice, we first examined whether the absence of GILZ is associated with enhanced susceptibility to $\mathrm{CCl}_{4}$-induced liver injury and cell death. Histological analysis of tissue sections did not reveal morphological differences in livers of WT and GILZ KO mice treated with oil or $\mathrm{CCl}_{4}$ (data not shown). Moreover, we compared the expression of several apoptosis-related genes in livers of control or $\mathrm{CCl}_{4}$-treated WT and GILZ $\mathrm{KO}$ mice. qPCR analysis revealed that mRNA expression levels of Fas (Fig. S2A), Fasl (Fig. S2B) and Bcl2l1 (Fig. S2C) did not significantly differ in livers of WT and GILZ KO mice. Thus, we did not find evidence of differences in initial $\mathrm{CCl}_{4}$-induced cell death between WT and GILZ KO mice.

Since leukocytes play a role in the initiation of inflammatory processes, HSC activation and LF progression ${ }^{57}$, and since GILZ has been implicated in the regulation of leukocytes trafficking ${ }^{34}$, we hypothesized that GILZ regulates leukocytes recruitment to liver at the initial stages of LF development. We evaluated the number of leukocytes in livers of WT and GILZ KO mice $72 \mathrm{~h}$ after treatment with oil or $\mathrm{CCl}_{4}$. As expected, $\mathrm{CCl}_{4}$ treatment led to a significant increase in the number of leukocytes in livers of WT mice, as compared to control mice (Fig. $3 \mathrm{~A}$ ). This initial $\mathrm{CCl}_{4}$ induced recruitment was significantly enhanced in GILZ $\mathrm{KO}$ mice (Fig. 3A). The increased leukocyte presence in 
A
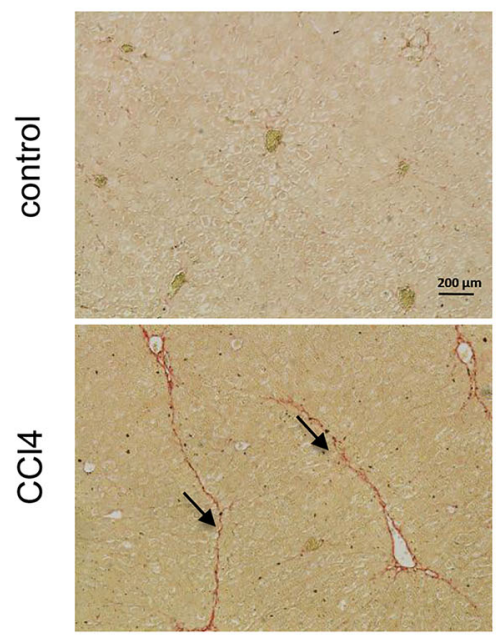

C

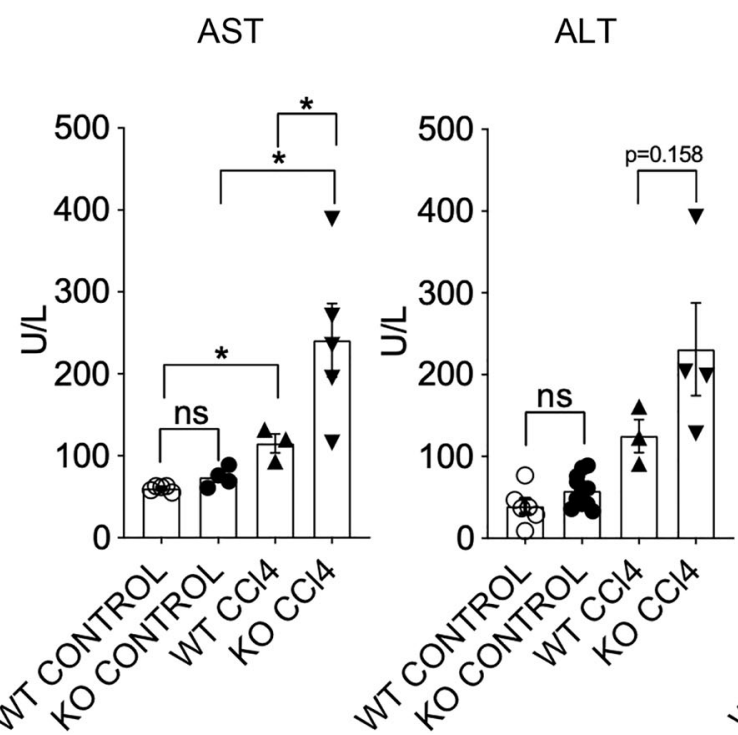

$\mathrm{KO}$

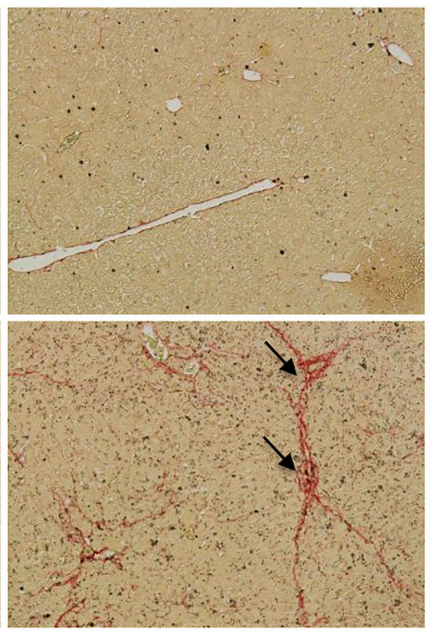

$\mathbf{E}$
B

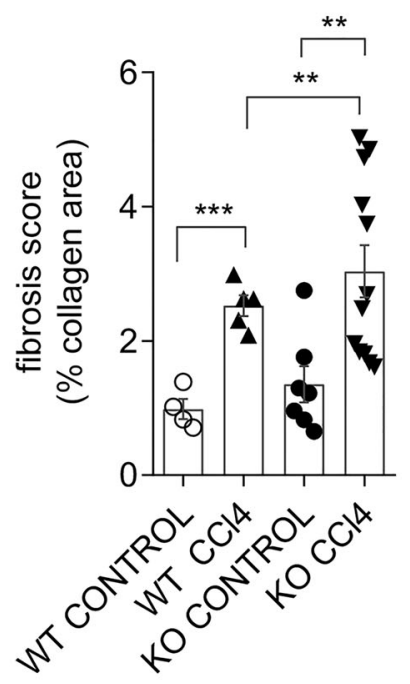

$\mathbf{F}$

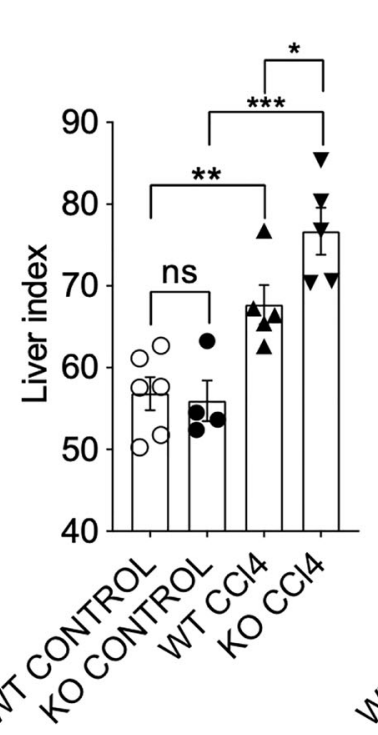

Asma

Fig. 1 Lack of GILZ results in increased $\mathrm{CCl}_{4}$-induced liver fibrosis development. A Sirius Red staining of liver sections from 4-month-old WT and GILZ KO mice that had been treated with oil (control; upper panels) or $\mathrm{CCl}_{4}$ (lower panels) for 6-7 weeks. Arrows indicate the areas of collagen deposition. B Graph shows the quantitative measurement of collagen deposition in all experimental groups. C, D AST (C) and ALT (D) levels in blood from 4-month-old WT and GILZ KO mice that had been treated with $\mathrm{CCl}_{4}$ for 6-7 weeks. E LI of 4-month-old WT and GILZ KO mice that had been treated with oil (control) or CCl4 for 6-7 weeks. F qPCR analysis of Asma mRNA expression in livers from 4-month-old WT and GILZ KO mice that had been treated for 6-7 weeks with oil (control) or $\mathrm{CCl}_{4}$. All data are presented relative to Actb mRNA expression. Each dot represents an individual mouse; bars indicate the mean. Results are presented as the means \pm SEM of three $(\mathbf{A}, \mathbf{B}, \mathbf{F})$ or two $(\mathbf{C}-\mathbf{E})$ independent experiments $\left({ }^{*} p<0.05\right.$, ${ }^{* * *} p<0.001$, ns not significant).

liver persisted also long term in GILZ KO mice treated with $\mathrm{CCl}_{4}$ for 6-7 weeks, as compared to similarly treated WT mice (Fig. 3B). These results indicate that the lack of GILZ is associated with enhanced leukocyte presence in the liver following $\mathrm{CCl}_{4}$-induced liver damage, and suggest that GILZ contributes to the regulation of the inflammatory process during LF development.
Lack of GILZ results in increased recruitment of monocytes and $\mathrm{CD} 4+\mathrm{T}$ cells to liver

In order to identify the immune cell subpopulations recruited to liver upon $\mathrm{CCl}_{4}$ treatment in WT and GILZ $\mathrm{KO}$ mice, we have performed flow cytometry analysis to evaluate the number of liver infiltrating monocytes $\left(\mathrm{Mac1}^{+} \mathrm{Gr}^{-}\right)$, granulocytes $\left(\mathrm{Mac1}^{+} \mathrm{Gr}^{+}\right)$, NK $\left(\mathrm{CD} 49 \mathrm{~d}^{+}\right)$, 
A
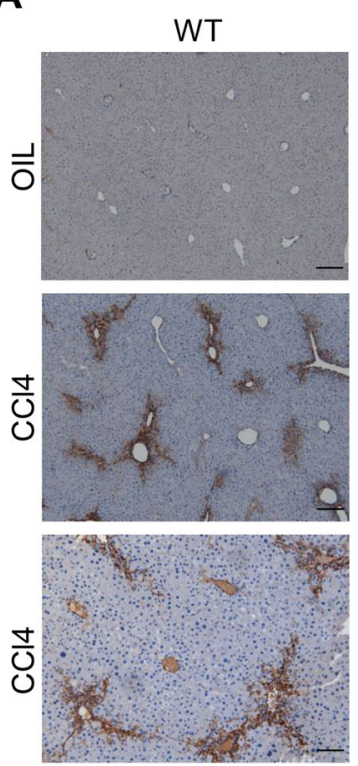

B
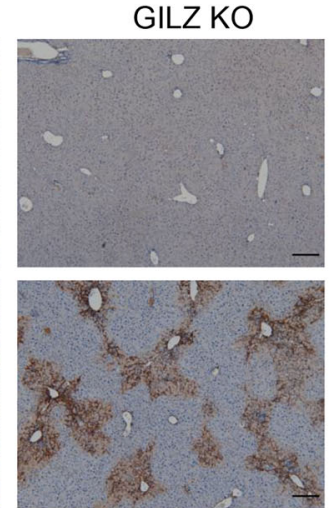

is
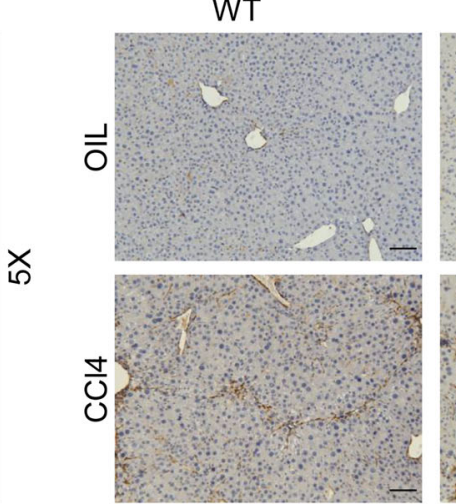

GILZ KO

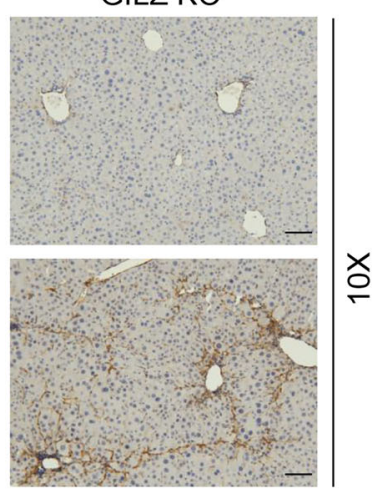

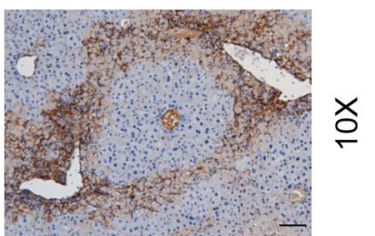

Fig. 2 GILZ deficiency is associated with elevated HSC activation. A, B Immunohistochemical analysis of a-SMA expression in the liver sections of WT and GILZ KO mice that had been treated with oil or $\mathrm{CCl}_{4}$ for $72 \mathrm{~h}(\mathbf{A})$ or 7 weeks (B). Original magnification, $5 \times$ or $10 \times$, as indicated. Arrows indicate the areas of a-SMA positivity. Scale bar, $100 \mu \mathrm{m}(5 \times$ magnification images) or $200 \mu \mathrm{m}$ (10× magnification images).

\section{A}

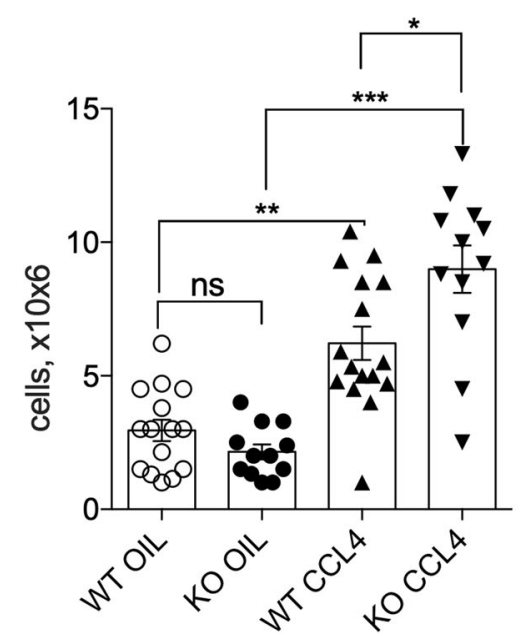

B

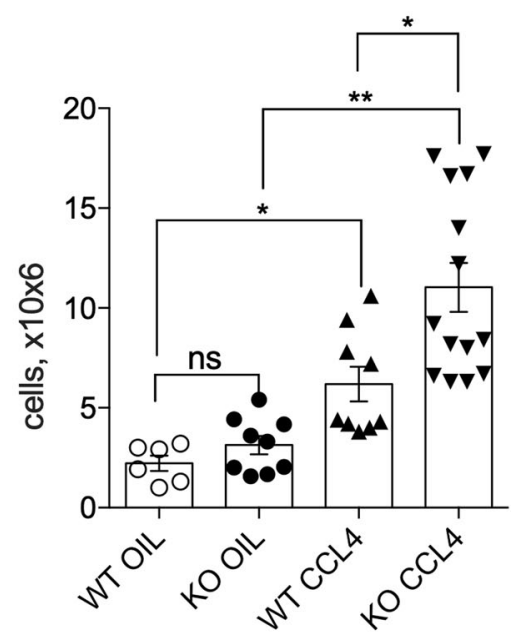

Fig. 3 Lack of GILZ results in increased leukocyte infiltration in the liver upon $\mathrm{CCl}_{4}$ treatment. A, B Number of infiltrated leukocytes in livers from 4-month-old WT and GILZ KO mice that had been treated with oil (control) or $\mathrm{CCl}_{4}$ for $72 \mathrm{~h}(\mathbf{A})$ and 6-7 weeks (B). Results are presented as the means \pm SEM. Each dot represents an individual mouse; bars indicate the mean. Data were pooled from five (A) or three (B) independent experiments $\left({ }^{*} p<0.05,{ }^{* *} p<0.005,{ }^{* *} p<0.001,{ }^{* * *} p<0.0001\right.$, ns not significant).

NKt $\left(\mathrm{CD} 49 \mathrm{~d}^{+} \mathrm{CD} 3 \mathrm{e}^{+}\right)$, and $\mathrm{T}$ cells $\left(\mathrm{CD} 3 \mathrm{e}^{+}\right.$; Fig. S3). Number of $\mathrm{CD}^{+} \mathrm{T}$ cells in liver showed a tendency increase in GILZ KO mice compared to similarly treated WT mice (Fig. S3A). However, if $\mathrm{CD}^{+} \mathrm{T}$ cells were further subdivided into $\mathrm{CD} 4^{+}$and $\mathrm{CD} 8^{+}$cells, we detected a significant increase in the number of $\mathrm{CD} 4^{+}$cells in livers derived from $\mathrm{CCl}_{4}$-treated GILZ $\mathrm{KO}$ mice compared to those derived from similarly treated WT mice (Fig. 4A). Instead, the number of $\mathrm{CD}^{+}$cells did not significantly differ between WT and GILZ KO livers (Fig. S4B). Since $\mathrm{Mac1}^{+}$cells represent the predominant subset in early hepatic lesions ${ }^{58}$, we compared the number 
A

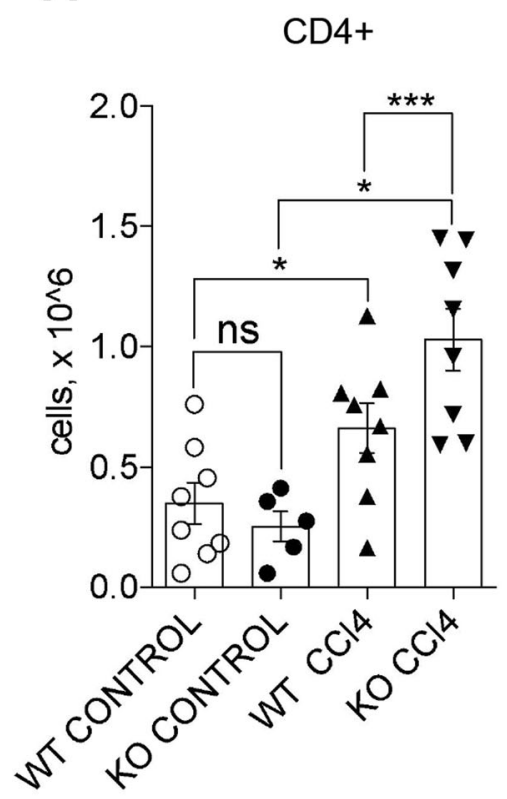

B

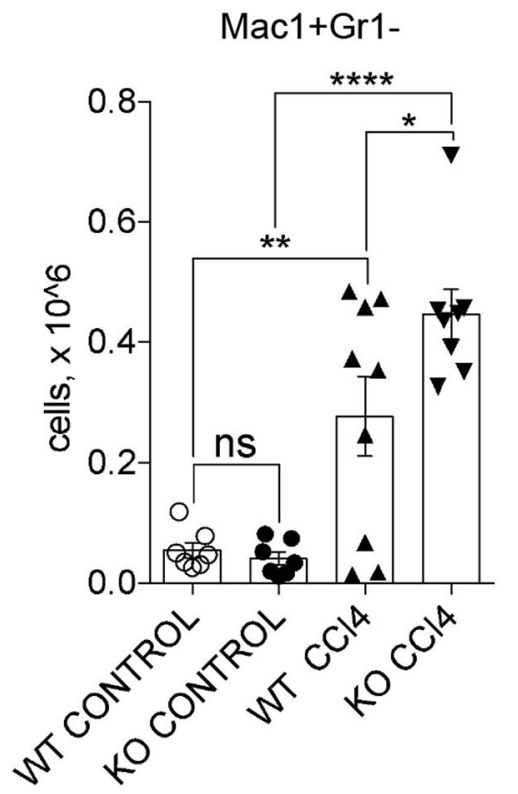

C

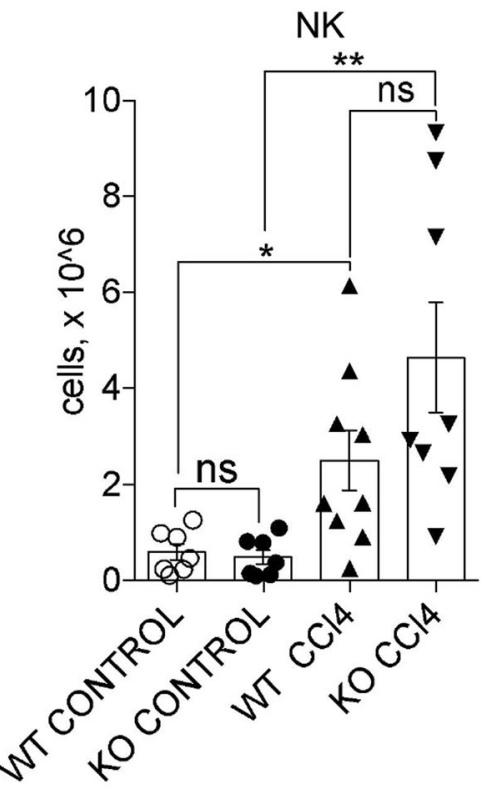

Fig. 4 Lack of GILZ results in increased monocyte, $\mathbf{C D} 4^{+} \mathbf{T}$ cell, and NK cell recruitment to the liver. $\mathbf{A}-\mathbf{C ~ N u m b e r ~ o f ~} C D 4^{+}(\mathbf{A}), \mathrm{MaC1}^{+} \mathrm{Gr} 1^{-}(\mathbf{B})$, and NK $(\mathbf{C})$ cells infiltrated in the livers of 4-month-old WT and GILZ KO mice that had been treated with oil (control) or CCl for $72 \mathrm{~h}$. Data were pooled from three independent experiments. Results are presented as the means \pm SEM; each dot represents an individual mouse; scale bars indicate the mean $\left({ }^{*} p<0.05,{ }^{* *} p<0.005{ }^{* * *} p<0.001,{ }^{* * *} p<0.0001\right.$, ns not significant).

of granulocytes $\left(\mathrm{Mac1}^{+} \mathrm{Gr} 1^{+}\right)$and monocytes/macrophages $\left(\mathrm{Mac}^{+} \mathrm{Gr}^{-}\right)$present in the liver upon $72 \mathrm{~h} \mathrm{CCl}_{4}$ treatment. The number of $\mathrm{Mac1}^{+} \mathrm{Gr}^{+}$cells did not significantly differ between WT and GILZ KO mice (Fig. S4C). Instead, the number of $\mathrm{Mac}^{+} \mathrm{Gr} 1^{-}$cells was significantly increased in livers of $\mathrm{CCl}_{4}$-treated GILZ KO mice compared to similarly treated WT mice (Fig. 4B). Moreover, GILZ deletion also mildly affected the number of $\mathrm{NK}$ cells in the livers of $\mathrm{CCl}_{4}$-treated mice, which revealed tendency increase in GILZ KO compared to similarly treated WT mice (Fig. 4C). NKt cells did not differ in their ability to infiltrate liver in WT and GILZ KO mice upon $\mathrm{CCl}_{4}$ treatment (Fig. S4D). Interestingly, the increase in $\mathrm{CD}^{+}{ }^{+}$cells in the livers of GILZ KO mice was associated with a significant increase in the mRNA expression of IL-4 cytokine in both early and late stages of LF development (Fig. S5A, B), and a modest decrease in IFN- $\gamma$ mRNA expression at the early stage (Fig. S5C), suggesting a predominance of the Th2-type response in the livers of mice lacking GILZ. To confirm that GILZ regulates recruitment of these leukocyte subpopulations to liver, we have used GILZ TG mice ${ }^{47}$. These mice presented a fourfold increase in GILZ protein levels in livers (Fig. S6A, B), which was sufficient to significantly suppress liver infiltration of T, NK cells, and macrophages in $\mathrm{CCl}_{4}$-treated GILZ TG mice compared to similarly treated WT mice (Fig. S6C-E). Altogether these results indicate that GILZ regulates the recruitment of monocytes, $\mathrm{T}$ and $\mathrm{NK}$ cells to livers challenged with $\mathrm{CCl}_{4}$.

\section{Lack of GILZ results in increased CCL2 expression}

Since liver injury leads to an upregulated expression of various chemokines ${ }^{59}$, and since GILZ controls the expression of several pro-inflammatory mediators, including chemokines ${ }^{22}$, we checked the mRNA expression levels of different chemokines and their receptors involved in fibrotic progression in the livers of WT and GILZ KO mice following $\mathrm{CCl}_{4}$ treatment. No significant differences in mRNA expression of Cxcl12 (Fig. S7A), Ccl5 (Fig. S7B), and its receptor Ccr5 (Fig. S7C) were found in livers of similarly treated WT and GILZ KO mice. qPCR analysis has revealed that $C c l 2$ mRNA expression was significantly increased in livers of WT mice upon $\mathrm{CCl}_{4}$ treatment (Fig. 5A), confirming that its expression is modulated upon LF induction. Interestingly, GILZ KO mice exhibited a significantly higher induction of $\mathrm{Ccl} 2 \mathrm{mRNA}$ expression compared to similarly treated WT mice (Fig. 5A). The mRNA expression levels of CCL2 receptor CCR2 did not significantly differ in livers of WT and GILZ KO mice, both at steady state and upon $\mathrm{CCl}_{4}$ treatment (Fig. 5B). These results demonstrate that GILZ controls the expression of CCL2 chemokine during hepatic injury. Consistent with previous experimental evidences $^{22}$, we detected a tendency increase in the levels 
A

ccl2 (CCl4 72h)

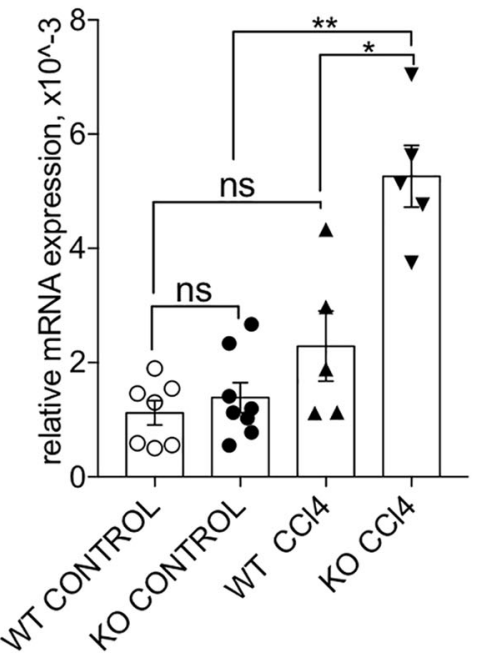

C

$\operatorname{ccr} 2(\mathrm{CCl} 472 \mathrm{~h})$

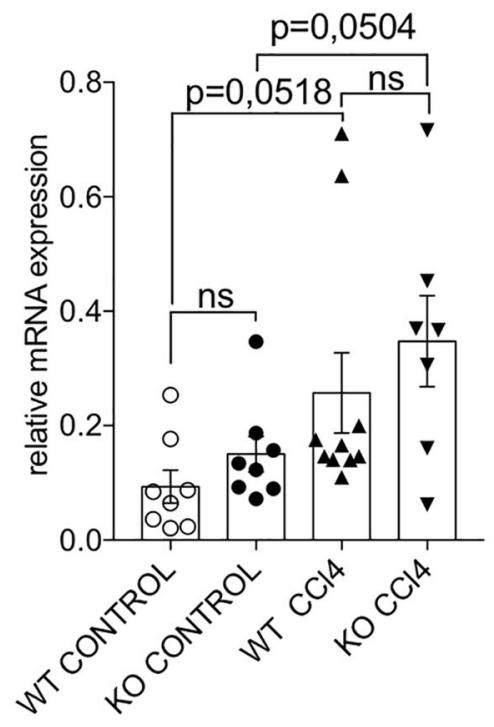

B

$c c / 2(\mathrm{CCl} 46-7-\mathrm{w})$
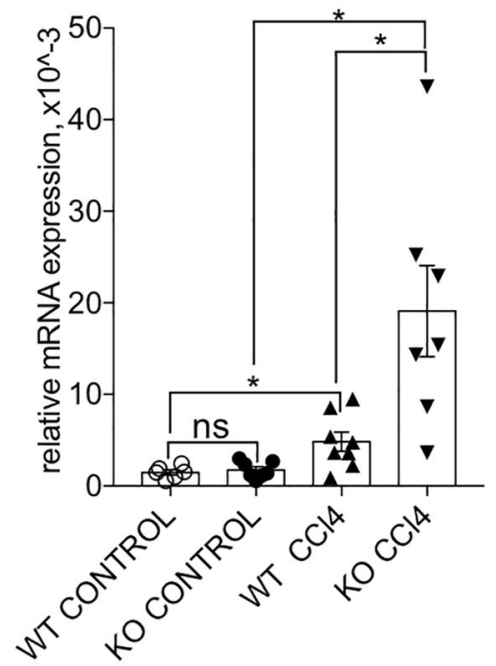

D

$\operatorname{ccr} 2(\mathrm{CCl} 46-7-\mathrm{w})$

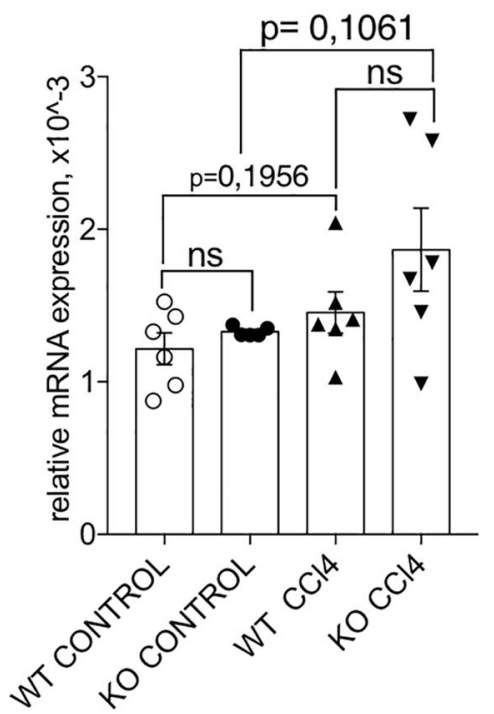

Fig. 5 Lack of GILZ results in increased $\mathrm{Ccl} 2 \mathrm{mRNA}$ expression in the liver following $\mathrm{CCl}_{4}$ treatment. A, B qPCR analysis of $C \mathrm{Cl} 2 \mathrm{mRNA}$ expression in the livers of 4-month-old WT and GILZ KO mice that had been treated for $72 \mathrm{~h}(\mathbf{A})$ or 6-7 weeks (B) with oil (control) or CCl 4 . C, $\mathbf{D}$ qPCR analysis of Ccr2 mRNA expression in the livers of 4-month-old WT and GILZ KO mice that had been treated for $72 \mathrm{~h}$ (C) or 6-7 weeks (D) with oil (control) or $\mathrm{CCl}_{4}$. All data are presented relative to Actb mRNA expression. Each dot represents an individual mouse; scale bars indicate the mean. Results are presented as the means \pm SEM. Data were pooled from three $(\mathbf{A}-\mathbf{C})$ or two (B-D) independent experiments $\left({ }^{*} p<0.05,{ }^{* *} p<0.005\right.$, ns not significant).

of phosphorylated ERK in the livers of $\mathrm{CCl}_{4}$-treated GILZ KO mice, compared to similarly treated WT mice, suggesting that deregulation of MAPK/ERK pathway might be related to the activation of $\mathrm{CCl} 2$ expression in the absence of GILZ (Fig. S8A, B).
CCR2 inhibition reverts enhanced $\mathrm{CCl}_{4}$-induced leukocytes infiltration into the liver in GILZ KO mice

In order to demonstrate that the augmented recruitment of $\mathrm{CD}^{+}{ }^{+} \mathrm{T}$ cells and $\mathrm{Mac1}^{+}$cells observed in GILZ KO mice depends on elevated CCL2 expression, we 
performed in vivo silencing of the CCL2 receptor CCR2 in WT and GILZ KO mice, and evaluated the number of infiltrating leukocytes in the livers following $\mathrm{CCl}_{4}$ treatment. The pharmacological downregulation of CCR2 expression was performed in vivo using siRNA embedded in LNPs $^{60}$. This system provides an efficient and clinically approved solution for gene silencing in vivo. The common issue with siRNA is miRNA-like activity that influence on the expression of partially complementary $\mathrm{mRNA}^{61}$. To avoid the contribution of off-target effects on the results, we selected several siRNA sequences targeting different CCR2 mRNA sites, introduced 2'-O-methyl pyrimidines and phosphorothioates to increase specificity and decrease immune response, and used the control siRNA with the same pattern of chemical modifications and same LNPs. The high efficacy of siRNA also decreases the possibility of off-target effects. Such setup provides a solid solution for proof-of-concept studies in the liver in vivo ${ }^{52,62}$. In order to achieve silencing of CCR2 mRNA, we compared previously published CCR2-targeting siRNA sequence with the best scored six siRNA in RAW264.7 cells (Supplementary Table 1). The most potent siRNAs ("siCCR2") was used in further in vivo studies. Experimental scheme for the in vivo CCR2 silencing and $\mathrm{CCl}_{4}$ treatment experiment is shown in Fig. S9A. Biweekly in vivo administration of LNP siCCR2 siRNA $(0.5 \mathrm{mg} / \mathrm{kg})$ led to a significant reduction in the levels of expression of Ccr2 mRNA (Fig. S9B). WT and GILZ KO mice were pretreated with CCR2 siRNA or Luciferase siRNA (siLUC, control) and then treated with $\mathrm{CCl}_{4}$ or oil. Evaluation of leukocyte number in the livers at $72 \mathrm{~h}$ after $\mathrm{CCl}_{4}$ treatment revealed that the downregulation of $\mathrm{Ccr} 2$ in a lack of significant differences in the leukocyte number observed in WT and GILZ KO mice (Fig. 6A). Importantly, this also led to a diminished HSC activation as evidenced by immunostaining for $\alpha$-SMA (Fig. $6 \mathrm{~B}$ ), suggesting that diminishing leukocytes recruitment also dampens the enhanced pro-fibrotic HSC activation observed in GILZ $\mathrm{KO}$ mice. Altogether these data demonstrate that the mechanisms underlying the enhanced recruitment of leukocytes in the livers of GILZ $\mathrm{KO}$ mice involve CCR2-CCL2 pathway.

\section{GILZ is downregulated in human patients with liver fibrosis}

In order to address the clinical relevance of GILZ-CCL2 axis in human LF, we analyzed GILZ mRNA expression in RNAseq data obtained from NASH and NAFLD patient liver samples ${ }^{63,64}$. We found that the mRNA expression of TSC22D3 gene encoding for GILZ in humans was significantly lower in NASH patients with a fat score $\geq 70$ compared to the healthy control and healthy obese patients group (Fig. 7A). The TSC22D3 mRNA expression was also significantly lower in NASH patients presenting LF (stages 1-4), as compared to those without fibrosis (Fig. 7B). Interestingly, in an independent cohort of NAFLD patients ${ }^{58}$, the $t s c 22 d 3$ mRNA
A
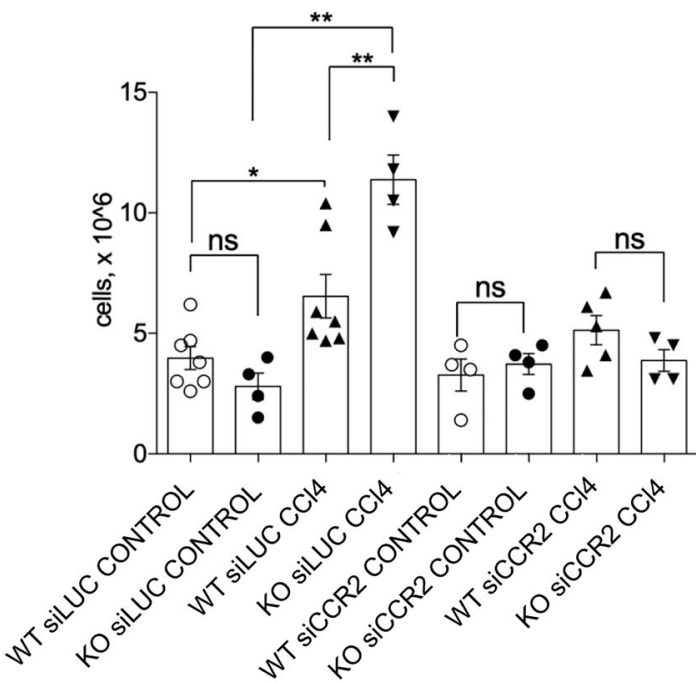

B
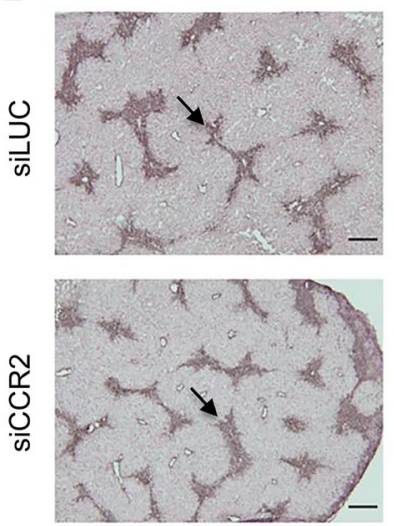

KO
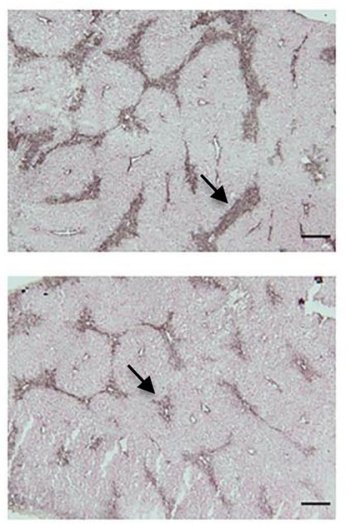

Fig. 6 Silencing CCR2 reverts enhanced leukocyte infiltration in GILZ KO mouse livers. A The number of leukocytes isolated from the livers of 4-month-old WT and GILZ KO mice that had been pretreated with siCCR2 or siLUC (LUC) for 1 week and then treated with oil (control) or CCl 4 for $72 \mathrm{~h}$. B Immunohistochemical analysis of a-SMA expression in the liver sections of WT and GILZ KO mice that had been pretreated with siLUC or siCCR2, and then treated with oil or $\mathrm{CCl}_{4}$ for $72 \mathrm{~h}$. Arrows indicate the areas of a-SMA positivity. Original magnification, 5x; scale bar, $200 \mu \mathrm{M}$. Results are presented as the means \pm SEM. Each dot represents an individual mouse; scale bars indicate the mean. Data were pooled from two independent experiments (** $p<0.005$, ns not significant). 

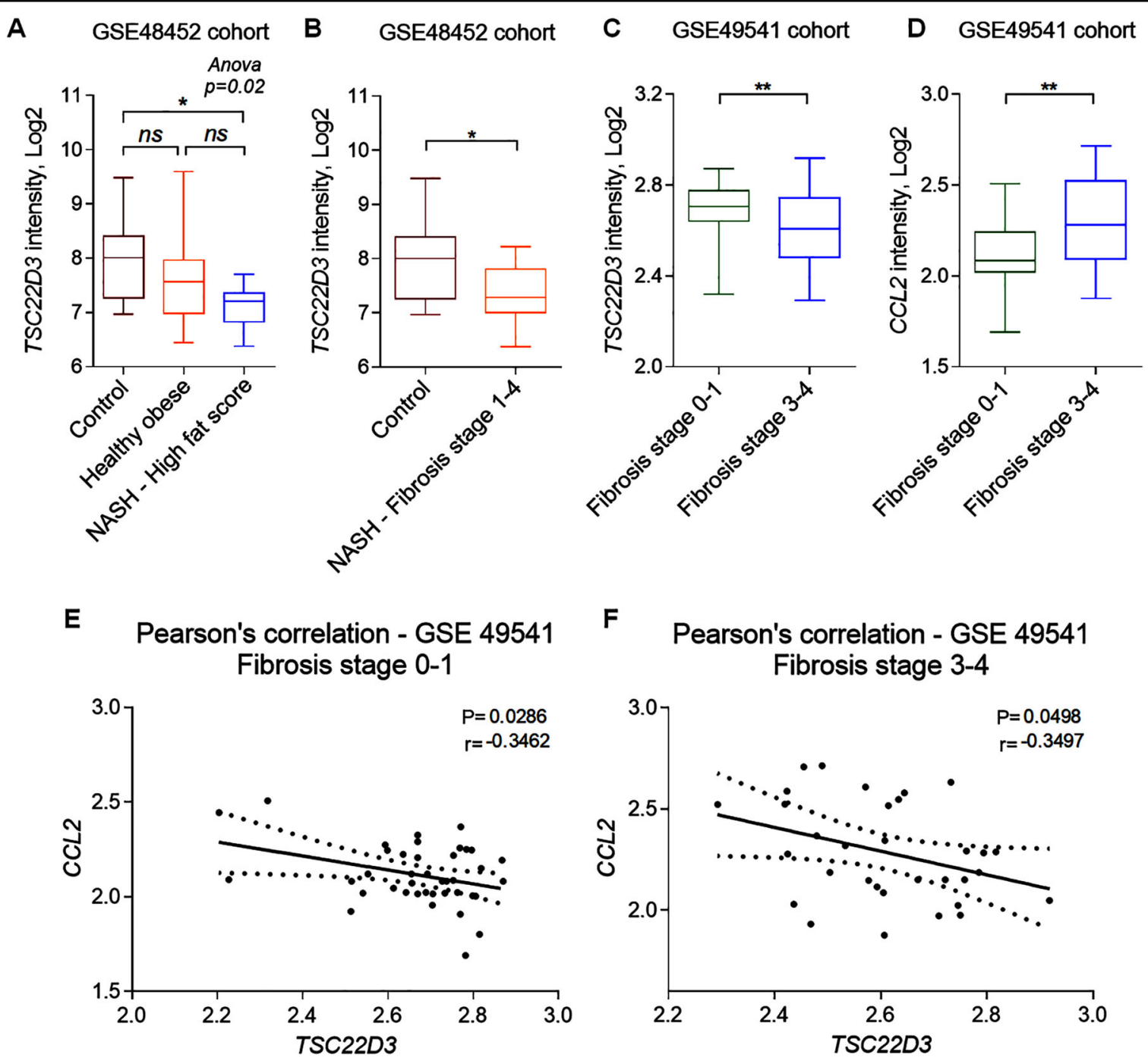

Fig. 7 GILZ is downregulated in patients with LF. TSC22D3 and CCL2 mRNA expression was analyzed in liver samples from NAFLD patients, according to GSE48452 (NASH patients) and GSE49541 (NAFLD patients) cohorts accessed in Gene Expression Omnibus. A, B TSC22D3 mRNA expression in NASH patients. Samples were classified as $\mathbf{A}$ high fat score when presenting a score of $\geq 70(n=9)$ and $\mathbf{B}$ fibrosis stages $1-4$, representing the samples in this score range $(n=14)$. C, D TSC22D3 (C) and CCL2 (D) gene expression in NAFLD patients with mild (stages $0-1 ; n=$ 40) and advanced (stages 3-4; $n=34$ ) fibrosis. E, F Pearson correlation between TSC22D3 and CCL2 mRNA expression in NAFLD patients. E Pearson correlation coefficient in NAFLD patients with mild (stages $0-1$ ) and (F) advanced (stages 3-4) fibrosis. Data are presented as a box and whiskers plot. ${ }^{*} p<0.05,{ }^{* *} p<0.01$.

expression inversely correlated with the degree of LF, as it was significantly lower in patients with advanced fibrosis (stages 3-4) compared to patients presenting fibrosis stages 0-1 (Fig. 7C; GSE49451). Conversely to decreased TSC22D3 mRNA levels, the mRNA expression of CCL2 was significantly higher in livers of NAFLD patients with higher fibrosis score compared to those with lower fibrosis score (Fig. 7D). Pearson correlation test confirmed that the mRNA expression levels of TSC22D3 and of $C C L 2$ were inversely correlated, as evidenced by a significant negative correlation scores both in samples with lower (Fig. 7E) and higher (Fig. 7F) fibrosis stages.
Altogether, these data demonstrate that GILZ expression is lower in livers of human NASH and NAFLD patients presenting fibrosis compared to healthy controls, and that its expression inversely correlates with the expression of CCL2.

\section{Discussion}

LF is associated with infectious, autoimmune, alcoholic, and metabolic liver diseases ${ }^{65}$. Current LF therapies include strategies based on inhibition of collagen synthesis, HSC-targeted therapies, and cytokine-blocking strategies. None of anti-fibrotic treatments have succeeded so 
$\mathrm{far}^{66}$. Thus, finding novel molecular players involved in LF can provide an important information for future drug development. GC have been applied to the clinical treatment of liver failure, alcoholic, and autoimmune hepatitis for many years ${ }^{15-18,67}$. GC's contradictory results in the treatment of $\mathrm{LF}$ are possibly linked to their opposing effects on immune cells and $\mathrm{HSC}^{20}$. Chronic use of GC is accompanied by important side effects ${ }^{13,68}$. GC have been implicated in the pathogenesis of NAFLD due their metabolic effects on liver and fat tissues ${ }^{69}$. Therefore, new drugs are needed to replace GC in treatment of inflammatory diseases, including LF.

The function of GILZ in LF development has not been directly addressed yet. Using mouse model of genetic ablation of GILZ in all tissues we observed an increased $\mathrm{CCl}_{4}$-induced LF. The expression of cell death-related genes was not altered in GILZ KO mice, thus we concluded that the enhanced susceptibility to $\mathrm{CCl}_{4}$-induced LF depends on other cellular processes. LF development is strictly associated with inflammation ${ }^{4,70-72}$. We found that GILZ controls the leukocyte recruitment to the liver during initial and chronic phases of LF development, following $\mathrm{CCl}_{4}$-induced liver damage. This is consistent with the role of GILZ in regulation of leukocyte trafficking in different experimental mouse models, such as rheumatoid arthritis ${ }^{35,73}$, spinal cord injury ${ }^{74}$, and inflammatory bowel diseases ${ }^{44,75}$. The inflammatory reaction of the Th2 type mediated by the $\mathrm{CD} 4^{+} \mathrm{T}$ lymphocytes and macrophages expressing Th2 cytokines represents an important factor involved in the higher susceptibility to fibrosis ${ }^{6,76-79}$. Interestingly, we found that the deletion of GILZ is associated with an increased presence of CD4 ${ }^{+}$ $\mathrm{T}$ cells and macrophages in the livers of $\mathrm{CCl}_{4}$-treated mice. Moreover, the absence of GILZ was associated with increased IL-4 and a decreased IFN- $\gamma$ expression. These results suggest that GILZ deletion alters the Th1/Th2 balance during the $\mathrm{CCl}_{4}$-mediated inflammatory reaction in the livers, resulting in a predominant pro-fibrotic Th2 immune state. This finding is in contrast with previous finding of GILZ promoting the Th2 differentiation ${ }^{31}$, but is consistent with the fact that the lack of GILZ causes the Th2 shift during spinal cord injury ${ }^{74}$. These data demonstrate that GILZ regulates the type of the inflammatory reaction in the liver and suggest that the regulation of Th1/Th2 differentiation by GILZ might be tissue context-dependent.

Chemokine expression by resident liver cells promotes infiltration of monocytes/macrophages, NK cells, NKt cells, neutrophils, B cells, and T cells ${ }^{59,79,80}$. GILZ was previously shown to control the expression of CCL5 and CXCL12 chemokines ${ }^{27,34}$. Here, we found that $\mathrm{CCl}_{4}$ treated GILZ KO mice expressed higher levels of $\mathrm{Ccl} 2$. To the contrary, the expression of CCL5 and CXCL12 did not differ between the livers of WT and GILZ KO mice, suggesting that the regulation of chemokine expression by GILZ is context-dependent. Interestingly, in GILZ KO mice, we found significant increase in the recruitment of the cell populations whose trafficking is regulated by CCL2, namely, macrophages, $\mathrm{CD} 4^{+} \mathrm{T}$ cell, and in part, NK cells ${ }^{79,81}$. At the same time recruitment of granulocytes, NKt and $\mathrm{CD} 8^{+} \mathrm{T}$ cells did not significantly differ between WT and GILZ KO mice. Taken together these data demonstrates that the deletion of GILZ is associated with elevated CCL2 expression and the enhanced liver recruitment of CCL2-sensitive immune cells.

To demonstrate that enhanced leukocyte recruitment to liver in GILZ KO mice is dependent on CCL2 levels, we performed in vivo RNAi silencing of CCR2 receptor in the liver via in vivo administration of CCR2 siRNA in LNPs. This system was successfully employed to downregulate mRNA expression in vivo in mice and nonhuman primates $^{60}$, and similar siRNA-LNP were approved by Food and Drug Administration (FDA) and European Medicines Agency (EMA) for the treatment of human Hereditary Transthyretin Amyloidosis ${ }^{82,83}$. RNA therapeutics evolved a lot during last years - a number of oligonucleotide drugs were approved by FDA and EMA and many of them are at late stages of clinical trials. Importantly, our data reveal that pharmacological downregulation of CCR2 attenuated leukocytes recruitment into the liver upon $\mathrm{CCl}_{4}$ challenge, thus providing a preclinical evidence of the possible therapeutic venue for the modulation of LF.

Importantly, our findings were supported by clinical data. Using public gene expression datasets, we found that the TSC22D3 mRNA expression was lower in specific subgroups of human NAFLD patients presenting elevated LF scores ${ }^{63,64}$. Moreover, the expression of GILZ inversely correlated with that of CCL2 in human NAFLD patients, in line with the evidence we obtained in mice. These data suggest that GILZ-CCL2 axis may have clinical relevance for the development of human LF, and thus represents perspective therapeutic target. In conclusion, our findings highlight an important role of GILZ in the regulation of LF development and provide the basis for the development of novel therapeutics to treat inflammatory liver diseases.

\section{Acknowledgements}

We would like to thank Marta Febo and Tatiana Viana de Barros for help with figures preparation.

\section{Author details}

'Department of Medicine and Surgery, University of Perugia, Severi Place 1, 06132 Perugia, Italy. ${ }^{2}$ Laboratory of Immune Regeneration and Experimental Hematology, Department of Pediatric Hematology and Oncology, Bambino Gesù Children Hospital, Viale San Paolo 15, Roma 00146, Italy. ${ }^{3}$ Skolkovo Institute of Science and Technology, Bolshoy Boulevard 30b1, 121205 Moscow, Russia. ${ }^{4}$ Institute for Molecular Medicine Finland, Helsinki Institute of Life Science, University of Helsinki, Helsinki Fl-00014, Finland. ${ }^{5}$ Gastroenterology Research Unit, Department of Experimental and Clinical Biochemical Sciences; Center of Excellence for Research, Transfer and High Education, DENOthe, 
University of Florence, Florence 50139, Italy. ${ }^{6}$ Department of Chemistry, Lomonosov Moscow State University, 119992 Moscow, Russia. ${ }^{7}$ Department of Philosophy, Social Sciences and Education, Ermini Place 1, 06123 Perugia, Italy

\section{Author contributions}

S.F., P.S., Z.B., T.M., M.B., M.P., C.F., T.P., and A.G. performed the experiments and analyzed the results; T.Z. and S.P. provided the expertise and reagents, and discussed the results; T.Z. wrote the manuscript; C.R., G.M., and S.B. provided the reagents, and discussed the results; S.B. and O.B. supervised the project; O.B. conceived the project, supervised the experiments, and wrote the manuscript. All authors have read and corrected the manuscript.

\section{Funding}

This work was supported by the Italian Ministry of Research and Education (PRIN 2017XZMBYX) to S.B.

\section{Ethics statement}

The experiments with animals were performed according to Italian (D.M. 116.192), European (Official Journal of European Community 358/1, December 18, 1986), and American (Animal Welfare Assurance No. A5594-01, Department of Health and Human Services, Washington, DC) laws, and procedures were approved by Ethical Committee of the University of Perugia.

\section{Conflict of interest}

The authors declare no competing interests.

\section{Publisher's note}

Springer Nature remains neutral with regard to jurisdictional claims in published maps and institutional affiliations.

Supplementary information The online version contains supplementary material available at https://doi.org/10.1038/s41419-021-03704-w.

Received: 16 December 2020 Revised: 8 April 2021 Accepted: 12 April 2021 Published online: 29 April 2021

\section{References}

1. Bataller, R. \& Brenner, D. A. Liver fibrosis. J. Clin. Investig. 115, 209-218 (2005)

2. Henderson, N. C., Rieder, F. \& Wynn, T. A. Fibrosis: from mechanisms to medicines. Nature 587, 555-566 (2020).

3. Mitchell, C. et al. Dual role of CCR2 in the constitution and the resolution of liver fibrosis in mice. Am. J. Pathol. 174, 1766-1775 (2009).

4. Wick, G. et al. The immunology of fibrosis. Annu. Rev. Immunol. 31, 107-135 (2013).

5. Shi, Z., Wakil, A. E. \& Rockey, D. C. Strain-specific differences in mouse hepatic wound healing are mediated by divergent $T$ helper cytokine responses. Proc. Natl Acad. Sci. USA 94, 10663-10668 (1997).

6. Wynn, T. A. Fibrotic disease and the $\mathrm{T}(\mathrm{H}) 1 / \mathrm{T}(\mathrm{H}) 2$ paradigm. Nat. Rev. Immunol. 4, 583-594 (2004)

7. Marra, F. \& Tacke, F. Roles for chemokines in liver disease. Gastroenterology 147, 577-94 e1 (2014)

8. Karlmark, K. R. et al. Hepatic recruitment of the inflammatory Gr1+ monocyte subset upon liver injury promotes hepatic fibrosis. Hepatology 50, 261-274 (2009).

9. Miura, K., Yang, L., van Rooijen, N., Ohnishi, H. \& Seki, E. Hepatic recruitment of macrophages promotes nonalcoholic steatohepatitis through CCR2. Am. J. Physiol. Gastrointest. Liver Physiol. 302, G1310-G1321 (2012).

10. Seki, E. et al. CCR2 promotes hepatic fibrosis in mice. Hepatology 50, 185-197 (2009).

11. Galastri, S. et al. Lack of CC chemokine ligand 2 differentially affects inflammation and fibrosis according to the genetic background in a murine mode of steatohepatitis. Clin. Sci. 123, 459-471 (2012).

12. Cain, D. W. \& Cidlowski, J. A. Immune regulation by glucocorticoids. Nat. Rev. Immunol. 17, 233-247 (2017).

13. Ronchetti, S., Migliorati, G., Bruscoli, S. \& Riccardi, C. Defining the role of glucocorticoids in inflammation. Clin. Sci. 132, 1529-1543 (2018).

14. Slominski, R. M. et al. Extra-adrenal glucocorticoid biosynthesis: implications for autoimmune and inflammatory disorders. Genes Immun. 21, 150-168 (2020).
15. Wang, F. \& Wang, B. Y. Corticosteroids or non-corticosteroids: a fresh perspective on alcoholic hepatitis treatment. Hepatobiliary Pancreat. Dis. Int. 10, 458-464 (2011)

16. Yeoman, A. D. et al. Prognosis of acute severe autoimmune hepatitis (AS-AlH, the role of corticosteroids in modifying outcome. J. Hepatol. 61, 876-882 (2014).

17. Boumpas, D. T., Chrousos, G. P., Wilder, R. L., Cupps, T. R. \& Balow, J. E. Glucocorticoid therapy for immune-mediated diseases: basic and clinical correlates. Ann. Intern. Med. 119, 1198-1208 (1993).

18. Xue, R. \& Meng, Q. The management of glucocorticoid therapy in liver failure. Front. Immunol. 10, 2490 (2019).

19. Kwon, H. J., Won, Y. S., Park, O., Feng, D. \& Gao, B. Opposing effects of prednisolone treatment on T/NKT cell- and hepatotoxin-mediated hepatitis in mice. Hepatology 59, 1094-1106 (2014).

20. Kim, K. H., Lee, J. M., Zhou, Y., Harpavat, S. \& Moore, D. D. Glucocorticoids have opposing effects on liver fibrosis in hepatic stellate and immune cells. Mol. Endocrinol. 30, 905-916 (2016).

21. D'Adamio, F. et al. A new dexamethasone-induced gene of the leucine zipper family protects T lymphocytes from TCR/CD3-activated cell death. Immunity $\mathbf{7}$ 803-812 (1997).

22. Bereshchenko, O., Migliorati, G., Bruscoli, S. \& Riccardi, C. Glucocorticoidinduced leucine zipper: a novel anti-inflammatory molecule. Front. Pharm. 10 308 (2019).

23. Bruscoli, S. et al. Long glucocorticoid-induced leucine zipper (L-GILZ) protein interacts with ras protein pathway and contributes to spermatogenesis control. J. Biol. Chem. 287, 1242-1251 (2012).

24. Bruscoli, S. et al. Glucocorticoid-induced leucine zipper (GILZ) and long GILZ inhibit myogenic differentiation and mediate anti-myogenic effects of glucocorticoids. J. Biol. Chem. 285, 10385-10396 (2010).

25. Cappetta, D. et al. Deficit of glucocorticoid-induced leucine zipper amplifies angiotensin-induced cardiomyocyte hypertrophy and diastolic dysfunction. J. Cell. Mol. Med. 1, 217-228 (2021).

26. Hoppstadter, J. et al. The glucocorticoid-induced leucine zipper mediates statin-induced muscle damage. FASEB J. 34, 4684-4701 (2020).

27. Cheng, Q. et al. GILZ overexpression inhibits endothelial cell adhesive function through regulation of NF-kappaB and MAPK activity. J. Immunol. 191, 424-433 (2013).

28. Shi, X. et al. A glucocorticoid-induced leucine-zipper protein, GILZ, inhibits adipogenesis of mesenchymal cells. EMBO Rep. 4, 374-380 (2003).

29. Bruscoli, S. et al. Genomic and non-genomic effects of different glucocorticoids on mouse thymocyte apoptosis. Eur. J. Pharm. 529, 63-70 (2006).

30. Bruscoli, S. et al. Lack of glucocorticoid-induced leucine zipper (GILZ) deregulates B-cell survival and results in B-cell lymphocytosis in mice. Blood 126, 1790-1801 (2015).

31. Cannarile, L. et al. Increased GILZ expression in transgenic mice up-regulates Th-2 lymphokines. Blood 107, 1039-1047 (2006).

32. Esposito, E. et al. Glucocorticoid-induced leucine zipper (GILZ) over-expression in T lymphocytes inhibits inflammation and tissue damage in spinal cord injury. Neurotherapeutics 9, 210-225 (2012).

33. Bereshchenko, O. et al. GILZ promotes production of peripherally induced Treg cells and mediates the crosstalk between glucocorticoids and TGF-beta signaling. Cell Rep. 7, 464-475 (2014).

34. Berrebi, D., Bruscoli, S., Cohen, N., Foussat, A. \& Migliorati, G. Bouchet-Delbos L, et al. Synthesis of glucocorticoid-induced leucine zipper (GILZ) by macrophages: an anti-inflammatory and immunosuppressive mechanism shared by glucocorticoids and IL-10. Blood 101, 729-738 (2003).

35. Fan, $\boldsymbol{H}$. et al. Macrophage migration inhibitory factor inhibits the antiinflammatory effects of glucocorticoids via glucocorticoid-induced leucine zipper. Arthritis Rheumatol. 66, 2059-2070 (2014).

36. Hoppstadter, J. et al. Glucocorticoid-induced leucine zipper: a critical factor in macrophage endotoxin tolerance. J. Immunol. 194, 6057-6067 (2015).

37. Ronchetti, S., Migliorati, G. \& Riccardi, C. GILZ as a mediator of the antiinflammatory effects of glucocorticoids. Front. Endocrinol. 6, 170 (2015).

38. Cohen, N. et al. GILZ expression in human dendritic cells redirects their maturation and prevents antigen-specific T lymphocyte response. Blood 107, 2037-2044 (2006)

39. Benkhoucha, M. et al. Hepatocyte growth factor limits autoimmune neuroinflammation via glucocorticoid-induced leucine zipper expression in dendritic cells. J. Immunol. 193, 2743-2752 (2014).

40. Ayroldi, E. et al. Modulation of T-cell activation by the glucocorticoid-induced leucine zipper factor via inhibition of nuclear factor kappaB. Blood 98, 743-753 (2001). 
41. Ayroldi, E. et al. Glucocorticoid-induced leucine zipper inhibits the Rafextracellular signal-regulated kinase pathway by binding to Raf-1. Mol. Cell Biol. 22, 7929-7941 (2002).

42. Di Marco, B. et al. Glucocorticoid-induced leucine zipper (GILZ)/NF-kappaB interaction: role of GILZ homo-dimerization and C-terminal domain. Nucleic Acids Res. 35, 517-528 (2007).

43. Vago, J. P. et al. The role and effects of glucocorticoid-induced leucine zipper in the context of inflammation resolution. J. Immunol. 194, 4940-4950 (2015).

44. Bruscoli, S. et al. Glucocorticoid-induced leucine zipper inhibits interferongamma production in B cells and suppresses colitis in mice. Front. Immunol. 9, 1720 (2018)

45. Hamdi, H. et al. Glucocorticoid-induced leucine zipper: a key protein in the sensitization of monocytes to lipopolysaccharide in alcoholic hepatitis. Hepatology 46, 1986-1992 (2007)

46. Robert, O. et al. Decreased expression of the glucocorticoid receptor-GILZ pathway in Kupffer cells promotes liver inflammation in obese mice. J. Hepatol. 64, 916-924 (2016).

47. Carceller, E. et al. Overexpression of Glucocorticoid-induced Leucine Zipper (GILZ) increases susceptibility to Imiquimod-induced psoriasis and involves cutaneous activation of TGF-beta1. Sci. Rep. 6, 38825 (2016).

48. Carino, A. et al. Disruption of TFGbeta-SMAD3 pathway by the nuclear receptor SHP mediates the antifibrotic activities of BAR704, a novel highly selective FXR ligand. Pharm. Res. 131, 17-31 (2018).

49. Sorcini, D. et al. Wnt/beta-catenin signaling induces integrin alpha4beta1 in $\mathrm{T}$ cells and promotes a progressive neuroinflammatory disease in mice. J. Immunol. 199, 3031-3041 (2017)

50. Bereshchenko, $\mathrm{O}$. et al. Deficiency and haploinsufficiency of histone macroH2A1.1 in mice recapitulate hematopoietic defects of human myelodysplastic syndrome. Clin. Epigenetics 11, 121 (2019).

51. Leuschner, F. et al. Therapeutic siRNA silencing in inflammatory monocytes in mice. Nat. Biotechnol. 29, 1005-1010 (2011).

52. Leboeuf, D. et al. Downregulation of the Arg/N-degron pathway sensitizes cancer cells to chemotherapy in vivo. Mol. Ther. 28, 1092-1104 (2020).

53. Giannini, E. G., Testa, R. \& Savarino, V. Liver enzyme alteration: a guide for clinicians. CMAJ 172, 367-379 (2005).

54. Biagioli, M. et al. GPBAR1 functions as gatekeeper for liver NKT cells and provides counterregulatory signals in mouse models of immune-mediated hepatitis. Cell Mol. Gastroenterol. Hepatol. 8, 447-473 (2019).

55. Friedman, S. L. Cellular sources of collagen and regulation of collagen production in liver. Semin. Liver Dis. 10, 20-29 (1990).

56. Puche, J. E. et al. A novel murine model to deplete hepatic stellate cells uncovers their role in amplifying liver damage in mice. Hepatology $\mathbf{5 7}$ 339-350 (2013).

57. Holt, A. P., Salmon, M., Buckley, C. D. \& Adams, D. H. Immune interactions in hepatic fibrosis. Clin. Liver Dis. 12, 861-882 (2008).

58. Rasmussen, J. W. et al. Mac-1+ cells are the predominant subset in the early hepatic lesions of mice infected with Francisella tularensis. Infect. Immun. 74, 6590-6598 (2006).

59. Saiman, Y. \& Friedman, S. L. The role of chemokines in acute liver injury. Front. Physiol. 3, 213 (2012)

60. Love, K. T. et al. Lipid-like materials for low-dose, in vivo gene silencing. Proc. Natl Acad. Sci. USA 107, 1864-1869 (2010).

61. Jackson, A. L. et al. Widespread siRNA "off-target" transcript silencing mediated by seed region sequence complementarity. RNA 12, 1179-1187 (2006).
62. Smekalova, E. M. et al. In vivo RNAi-Mediated elF3m knockdown affects ribosome biogenesis and transcription but has limited impact on mRNAspecific translation. Mol. Ther. Nucleic Acids 19, 252-266 (2020).

63. Ahrens, M. et al. DNA methylation analysis in nonalcoholic fatty liver disease suggests distinct disease-specific and remodeling signatures after bariatric surgery. Cell Metab. 18, 296-302 (2013).

64. Moylan, C. A. et al. Hepatic gene expression profiles differentiate presymptomatic patients with mild versus severe nonalcoholic fatty liver disease. Hepatology 59, 471-482 (2014).

65. Roehlen, N., Crouchet, E. \& Baumert, T. F. Liver fibrosis: mechanistic concepts and therapeutic perspectives. Cells $\mathbf{9}, 4$ (2020).

66. Rockey, D. C. Current and future anti-fibrotic therapies for chronic liver disease Clin. Liver Dis. 12, 939-962 (2008). xi.

67. Mathurin, $\mathrm{P}$. et al. Corticosteroids improve short-term survival in patients with severe alcoholic hepatitis ( $\mathrm{AH}$, individual data analysis of the last three randomized placebo controlled double blind trials of corticosteroids in severe $\mathrm{AH}$. J. Hepatol. 36, 480-487 (2002).

68. Vandewalle, J., Luypaert, A., De Bosscher, K. \& Libert, C. Therapeutic mechanisms of glucocorticoids. Trends Endocrinol. Metab. 29, 42-54 (2018).

69. Woods, C. P., Hazlehurst, J. M. \& Tomlinson, J. W. Glucocorticoids and nonalcoholic fatty liver disease. J. Steroid Biochem. Mol. Biol. 154, 94-103 (2015).

70. Wick, G. et al. The immunology of fibrosis: innate and adaptive responses. Trends Immunol. 31, 110-119 (2010).

71. Heymann, F., Trautwein, C. \& Tacke, F. Monocytes and macrophages as cellular targets in liver fibrosis. Inflamm. Allergy Drug Targets 8, 307-318 (2009).

72. Chaudhry, S., Emond, J. \& Griesemer, A. Immune cell trafficking to the liver. Transplantation 103, 1323-1337 (2019).

73. Beaulieu, E. \& Morand, E. F. Role of GILZ in immune regulation, glucocorticoid actions and rheumatoid arthritis. Nat. Rev. Rheumatol. 7, 340-348 (2011).

74. Mazzon, E. et al. Glucocorticoid-induced leucine zipper (GILZ) controls inflammation and tissue damage after spinal cord injury. CNS Neurosci. Ther 20, 973-981 (2014)

75. Cannarile, L. et al. Glucocorticoid-induced leucine zipper is protective in Th1mediated models of colitis. Gastroenterology 136, 530-541 (2009).

76. Mahrouf-Yorgov, M. et al. Increased susceptibility to liver fibrosis with age is correlated with an altered inflammatory response. Rejuvenation Res. 14, 353-363 (2011).

77. Coutinho, H. M. et al. Th2 cytokines are associated with persistent hepatic fibrosis in human Schistosoma japonicum infection. J. Infect. Dis. 195, 288-295 (2007).

78. Barron, L. \& Wynn, T. A. Fibrosis is regulated by Th2 and Th17 responses and by dynamic interactions between fibroblasts and macrophages. Am. J. Physiol. Gastrointest. Liver Physiol. 300, G723-G728 (2011).

79. Heymann, F. \& Tacke, F. Immunology in the liver-from homeostasis to disease Nat. Rev. Gastroenterol. Hepatol. 13, 88-110 (2016).

80. Oo, Y. H., Shetty, S. \& Adams, D. H. The role of chemokines in the recruitment of lymphocytes to the liver. Dig. Dis. 28, 31-44 (2010).

81. Karlmark, K. R., Wasmuth, H. E., Trautwein, C. \& Tacke, F. Chemokine-directed immune cell infiltration in acute and chronic liver disease. Expert Rev. Gastroenterol. Hepatol. 2, 233-242 (2008).

82. Bauer, D. et al. Diallyl disulfide inhibits TNFalpha induced $C C L 2$ release through MAPKVERK and NF-Kappa-B signaling. Cytokine 75, 117-126 (2015).

83. Adams, D. et al. Patisiran, an RNAi therapeutic, for hereditary transthyretin amyloidosis. N. Engl. J. Med. 379, 11-21 (2018). 